\title{
Use of noninvasive volume assessment methods to predict acute blood loss in spontaneously breathing volunteers
}

\author{
Asim Tomo ${ }^{1}$, Murat Pekdemir ${ }^{2}$, Ibrahim Ulas Ozturan ${ }^{3}$, \\ Nurettin Ozgur Dogan², Elif Yaka², Serkan Yilmaz ${ }^{2}$ \\ 'Department of Emergency Medicine, Kadikoy Medicana Hospital, Istanbul, Turkey \\ ${ }^{2}$ Department of Emergency Medicine, Faculty of Medicine, Kocaeli University, Kocaeli, Turkey \\ ${ }^{3}$ Department of Emergency Medicine, Mersin Toros State Hospital, Mersin, Turkey
}

Objective The use of noninvasive volume assessment methods to predict acute blood loss in spontaneously breathing patients remains unclear. We aimed to investigate changes in the pleth variability index (PVI), vena cava collapsibility index ( $\mathrm{VCCl})$, end-tidal carbon dioxide (EtCO2), pulse pressure (PP), and mean arterial pressure (MAP) in spontaneously breathing volunteers after acute loss of $450 \mathrm{~mL}$ blood and passive leg raise (PLR).

Methods This prospective observational study enrolled healthy volunteers in the blood donation center of an academic hospital. We measured the PVI, EtCO2, VCCI, MAP, and PP before blood donation; at the 0th and 10th minute of blood donation; and after PLR. The primary outcome was the changes in PVI, EtCO2, VCCI, MAP, and PP.

Results We enrolled thirty volunteers. There were significant differences among the four obtained measurements of the PVI, EtCO2, and MAP $(\mathrm{P}<0.001, \mathrm{P}<0.001, \mathrm{P}<0.001$, respectively). Compared to the predonation values, post-hoc analysis revealed an increase in the PVI at the 0th min postdonation (mean difference [MD], 5.4 $\pm 5.9 ; 95 \%$ confidence interval $[\mathrm{Cl}],-7.6$ to -3.1 ; $\mathrm{P}<0.001)$; a decrease in the EtCO2 and MAP at the 0th and 10th minute postdonation, respectively (MD, $2.4 \pm 4.6 ; 95 \% \mathrm{Cl}, 0.019$ to $4.84 ; \mathrm{P}=0.008$ and $\mathrm{MD}, 6.4 \pm 6.4 ; 95 \% \mathrm{Cl}, 3$ to $9.7 ; \mathrm{P}<0.001$, respectively). Compared with EtCO2 at the 10th minute, the value increased after PLR (MD, $1.8 \pm 3.2 ; 95 \% \mathrm{Cl}, 0.074$ to $4.44 ; \mathrm{P}=0.006)$.

Conclusion The PVI and EtCO2 could detect early hemodynamic changes after acute blood loss. However, it remains unclear whether they can determine volume status in spontaneously breathing patients.

Keywords Photoplethysmography; Capnography; Hypovolemia; Shock

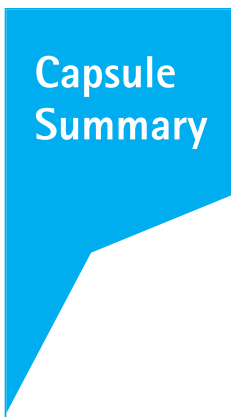

What is already known

The benefit of PVI, EtCO2, VCCI, MAP, and PP in detecting acute intravascular volume changes in mechanically ventilated patients was demonstrated. However, previous data on the accuracy of these methods in spontaneously breathing patients is limited.

What is new in the current study

PVI, EtCO2, and MAP are reliable methods for detecting acute blood loss in spontaneously breathing population.
elSSN: 2383-4625

Received: 4 March 2020

Revised: 4 May 2020

Accepted: 12 May 2020

Correspondence to: Ibrahim Ulas Ozturan

Department of Emergency Medicine, Mersin Toros State Hospital, Mesudiye, 5117. Sk. No:34, 33060 Akdeniz/Mersin, Turkey

E-mail: i.ozturan@saglik.gov.tr ORCID

https://orcid.org/0000-0002-1364-5292

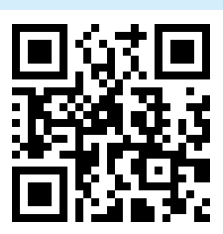

How to cite this article:

Tomo A, Pekdemir M, Ozturan IU, Dogan NO, Yaka E, Yilmaz S. Use of noninvasive volume assessment methods to predict acute blood loss in spontaneously breathing volunteers. Clin Exp Emerg Med 2021;8(1):9-15. https://doi.org/10.15441/ ceem. 20.021

This is an Open Access article distributed under the terms of the Creative Commons Attribution Non-Commercial License (https:// creativecommons.org/licenses/by-nc/4.0/). 


\section{INTRODUCTION}

Hypovolemia is among the most common causes of circulatory failure in critically ill patients. ${ }^{1}$ Early hypotension detection and determining its underlying reason could improve the outcome in the emergency department (ED). ${ }^{2}$ Generally, fluid resuscitation in patients with hypotension caused by hypovolemia can achieve an adequate response. However, it is often harmful in cases where the hypotension is not hypovolemia-related. ${ }^{3,4}$ Therefore, the volume status should be evaluated as an initial step in critically ill patients in the ED. There are several invasive methods for assessing volume status in mechanically ventilated patients under sedation in intensive care settings. ${ }^{5}$ However, a majority of these methods are not feasible for spontaneously breathing patients in the ED. ${ }^{6}$ Therefore, there is a need for fast, easy, and noninvasive methods for volume assessment in critically ill patients in the ED.

Photoplethysmography (PPG) is an optical technology employed in pulse oximeters. The perfusion index is the ratio between PPG signals of the pulsatile (arterial component) and non-pulsatile components (other tissues) in the pulse oximeter. The pleth variability index (PVI) is a dynamic indicator of the volume status, which can be determined by respiratory variations in the perfusion index. ${ }^{8}$ Previous studies have assessed the ability of the PVI value to predict fluid responsiveness in mechanically ventilated patients. ${ }^{9}$ However, the utility of the PVI in determining acute intravascular volume changes in spontaneously breathing patients remains unclear. Previous studies on the PVI in spontaneously breathing patients reported that it could weakly predict intravascular volume changes. ${ }^{8,10}$ However, hypovolemia induced by acute blood depletion was present in only a small number of the patients included in these studies. ${ }^{10}$

End-tidal carbon dioxide (EtCO2) is an important metabolism indicator that could be used to assess various conditions of critically ill patients, including volume status and responsiveness. ${ }^{11,12}$ EtCO2 utility in noninvasive monitoring of patients in shock has been reported ${ }^{13}$; however, there have been few studies on EtCO2 accuracy in early-stage hypovolemia. ${ }^{14,15}$

Previous studies have reported the role of the vena cava collapsibility index (VCCI) in volume status prediction. ${ }^{16}$ The VCCl has been demonstrated as an indirect indicator of central venous pressure with respect to volume status. ${ }^{5,6}$ However, its predictive ability of early phase acute volume depletion remains unclear. ${ }^{17}$ Further, the mean arterial pressure (MAP) and the pulse pressure (PP) are widely used noninvasive monitoring indexes derived from the arterial blood pressure (BP). Although these indexes have been suggested in hypovolemia monitoring ${ }_{1}^{18}$ their utility in the advanced shock stages remains unclear.
We aimed to investigate the utility of noninvasive volume assessment methods, including PVI, EtCO2, VCCl, MAP, and PP, in monitoring acute intravascular volume changes in healthy adults after 1-unit ( $450 \mathrm{~mL}$ ) blood donation and passive leg raise (PLR).

\section{METHODS}

\section{Study design and setting}

This prospective observational study was conducted in the blood center of an academic hospital that received an annual blood donation from 17,000 healthy volunteers (male 96.4\%) between June 2015 and July 2015. Institutional review board approval was obtained for the study (08.07.2014-15/12). All study participants provided written informed consent prior to study participation.

\section{Participant selection}

We enrolled all healthy blood donors who provided consent for study participation. We excluded volunteers unsuitable for blood donation (active infection, intravenous drug use, hemoglobin levels $<13 \mathrm{~g} / \mathrm{dL}$, current medication use, alcohol use within the last 24 hours). Moreover, we excluded volunteers with cardiac dysrhythmia or skin disruption, which may affect the study measurements.

\section{Study protocol}

We measured the PVI, EtCO2, VCCl, MAP, and PP with the patient in supine position before donation and at the 0th and 10th minute postdonation. Subsequently, the patients performed the 45-degree PLR (Fig. 1), and the aforementioned measurements were obtained after holding this position for 90 seconds. We obtained PVI and Sp02 measurements using the Radical-7 PPG device (Masimo Corp., Irvine, CA, USA). A pulse oximeter probe (LNOP Adt, Masimo Corp.) was attached on the 4th finger of the non-dominant hand. The pulse oximeter was connected to the Radical 7

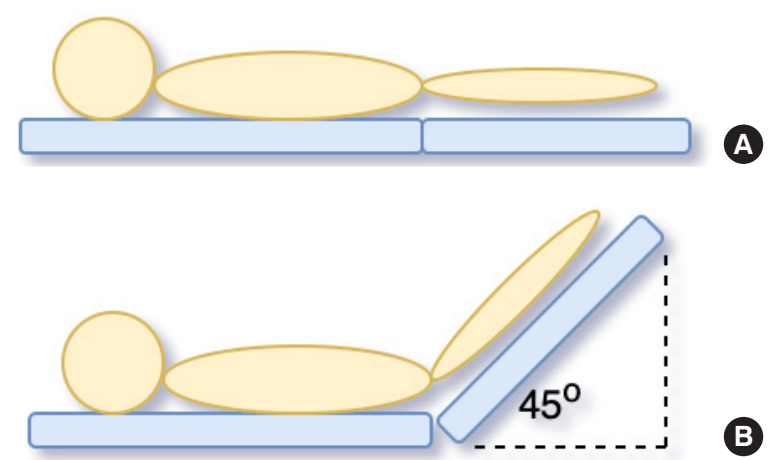

Fig. 1. Measurement positions. (A) Predonation, postdonation at the 0th and postdonation at the 10th minute measurements and (B) passive leg raise measurements. 
monitor (Masimo Corp.) with PVI software ver. 7.0.3.3. (Masimo Corp.).

We used the B-20 monitor (Mindray, Milwaukee, WI, USA) to measure the arterial $\mathrm{BP}$, respiratory rate, and $\mathrm{EtCO} 2$ using the sidestream method. We conducted side-stream EtCO2 measurements using a sampling port adapted to a simple oxygen mask. MAP and $\mathrm{PP}$ were calculated as follows: $\mathrm{PP}=$ systolic $\mathrm{BP}$-diastolic $\mathrm{BP}$ and $M A P=2 / 3$ diastolic $B P+1 / 3$ systolic $B P$.

$\mathrm{VCCl}$ measurements were obtained by an ultrasound-trained emergency physician using the Mylab Five (Esaote, Maastricht, the Netherlands) bedside ultrasound device and 1-8 MHz CA431 convex transducer.

\section{Outcome measures}

The primary outcomes were changes in PVI, EtCO2, MAP, VCCl, and $\mathrm{PP}$ at the 0th and 10th minute postdonation, as well as after the PLR.

\section{Statistical analysis}

We calculated the study sample size using G-Power for Mac OS X ver. 3.1.9.2 (Heinrich Heine University Dusseldorf, Dusseldorf, Germany). Considering four measurements from each volunteer, the estimated sample size was 30 with an assumed medium effect size of $f=0.25$, type- 1 error of 0.05 , and study power of $90 \%$. All statistical analyses were performed using IBM SPSS Statistics ver. 21.0 (IBM Corp., Armonk, NY, USA) and Matlab ver. R2019a (Mathworks, Natick, MA, USA). The Shapiro-Wilk test was used to assess the normality of the variables. Normally distributed data were expressed as mean with standard deviation and 95\% confidence interval (CI). Non-normally distributed data were expressed as median and interquartile range. Repeated-measures analysis of variance and Bonferroni-adjusted post hoc pairwise comparison were used to assess variations in the continuous variables. The Mauchly test was used to assess the validity of sphericity. We used Greenhouse-Geisser and Huynh-Feldt corrections if the epsilon was $<0.75$ and $>0.75$, respectively. Decimals in the results were rounded off. Statistical significance was set at $P<0.05$. For measurements that were assessed using the Bonferroni-adjusted post hoc test, statistical significance was set at $\mathrm{P}<0.008$.

\section{RESULTS}

We enrolled 30 volunteers with a median age of 30 (6) years and a median weight of 75 (10) kilograms. All the study participants were male since the vast majority of the blood donors were male (96.4\%). Table 1 shows changes in the hemodynamic, plethysmographic, and respiratory variables.

There were significant differences among all four PVI measurements $(F[2.520 ; 73.070]=10.162, P<0.001)$. Post hoc analysis revealed difference in the $\mathrm{PVI}$ at predonation (18.8\%) and at the 0th minute postdonation (24.2\%) (mean difference [MD], 5.4 \pm 5.9 ; $95 \% \mathrm{Cl}_{1}-7.6$ to $-3.1 ; \mathrm{P}<0.001$ ) (Fig. 2).

Further, there were significant changes in the mean values of EtCO2 and MAP: $(F[2.503 ; 72.577]=6.615 ; \mathrm{P}<0.001)$ and $(\mathrm{F}[1.960$; $56.832]=10.050 ; P<0.001)$, respectively. Post hoc analysis revealed a significant decrease in the $\mathrm{EtCO} 2$ at the 0th minute postdonation compared to the predonation value (MD, 2.4 \pm 4.6 ; 95\% $\mathrm{Cl}, 0.019$ to $4.84 ; \mathrm{P}=0.008$ ). Moreover, there was a significant in-

Table 1. Changes in hemodynamic, plethysmographic and respiratory variables of the participants

\begin{tabular}{|c|c|c|c|c|c|}
\hline & Predonation & Postdonation 0th min & Postdonation 10th min & PLR position & P-value \\
\hline \multicolumn{6}{|c|}{ Arterial blood pressure and heart rate } \\
\hline Pulse (bpm) & $75 \pm 11$ & $75 \pm 10$ & $74 \pm 9$ & $73 \pm 10$ & 0.374 \\
\hline $\mathrm{SBP}(\mathrm{mmHg})$ & $126 \pm 11$ & $120 \pm 11$ & $117 \pm 11$ & $119 \pm 9$ & $<0.001$ \\
\hline $\mathrm{DBP}(\mathrm{mmHg})$ & $67 \pm 7$ & $63 \pm 8$ & $62 \pm 6$ & $63 \pm 5$ & 0.002 \\
\hline $\mathrm{MAP}(\mathrm{mmHg})$ & $87 \pm 7$ & $82 \pm 8$ & $80 \pm 6$ & $81 \pm 5$ & $<0.001$ \\
\hline $\mathrm{PP}(\mathrm{mmHg})$ & $58 \pm 10$ & $57 \pm 8$ & $55 \pm 9$ & $55 \pm 8$ & 0.121 \\
\hline \multicolumn{6}{|l|}{ Pulse oximeter PPG } \\
\hline PVI (\%) & $18.8 \pm 6.4$ & $24.2 \pm 8.6$ & $25.1 \pm 8$ & $25.0 \pm 8.9$ & $<0.001$ \\
\hline $\mathrm{PI}(\%)$ & $4.17 \pm 2.7$ & $3.56 \pm 1.9$ & $3.9 \pm 1.9$ & $3.7 \pm 2.1$ & 0.453 \\
\hline $\mathrm{Sp02}(\%)$ & $97.8 \pm 1.4$ & $97.9 \pm 1.1$ & $98 \pm 1.1$ & $98 \pm 0.8$ & 0.611 \\
\hline \multicolumn{6}{|l|}{ Other } \\
\hline VCl-Cl (\%) & $37.5 \pm 16.1$ & $37.9 \pm 13.4$ & $33.6 \pm 15.4$ & $33.7 \pm 14.6$ & 0.395 \\
\hline $\mathrm{EtCO} 2(\mathrm{mmHg})$ & $33 \pm 5$ & $31 \pm 5$ & $30 \pm 5$ & $31 \pm 5$ & 0.001 \\
\hline $\mathrm{RR}$ (resp/min) & $18 \pm 3$ & $17 \pm 3$ & $17 \pm 2$ & $18 \pm 3$ & 0.085 \\
\hline
\end{tabular}

Values are presented as mean \pm standard deviation.

PLR, passive leg raise; SBP, systolic blood pressure; DBP, diastolic blood pressure; MAP, mean arterial pressure; PP, pulse pressure; PPG, photoplethysmography; PVI, pleth variability index; PI, perfusion index; SpO2, peripheral oxygen saturation; $\mathrm{VCl}-\mathrm{Cl}$, vena cava inferior collapsibility index; EtCO2, end-tidal carbon dioxide; RR, respiratory rate. 


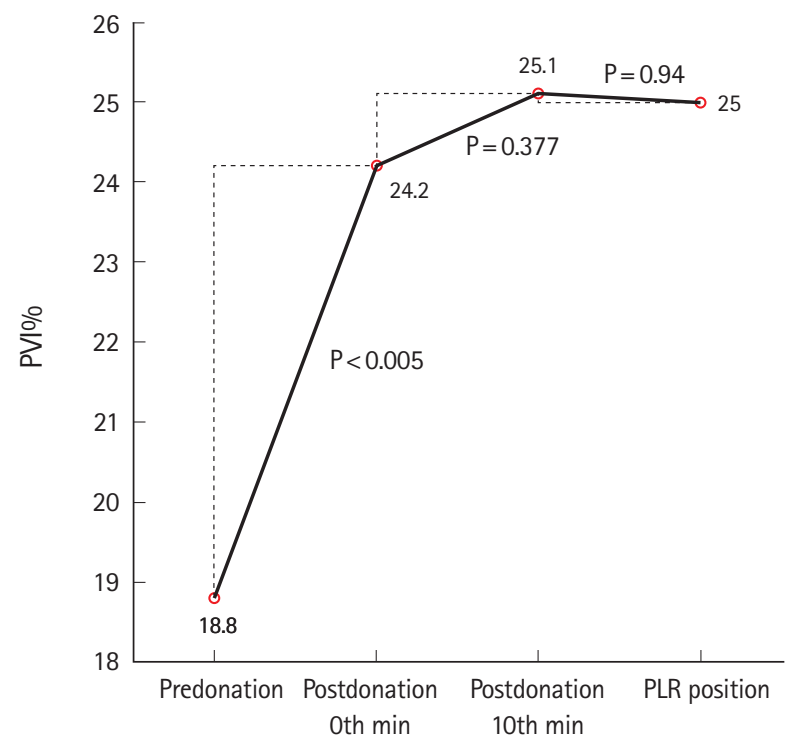

Fig. 2. Variations in pleth variability index (PVI). PLR, passive leg raise.

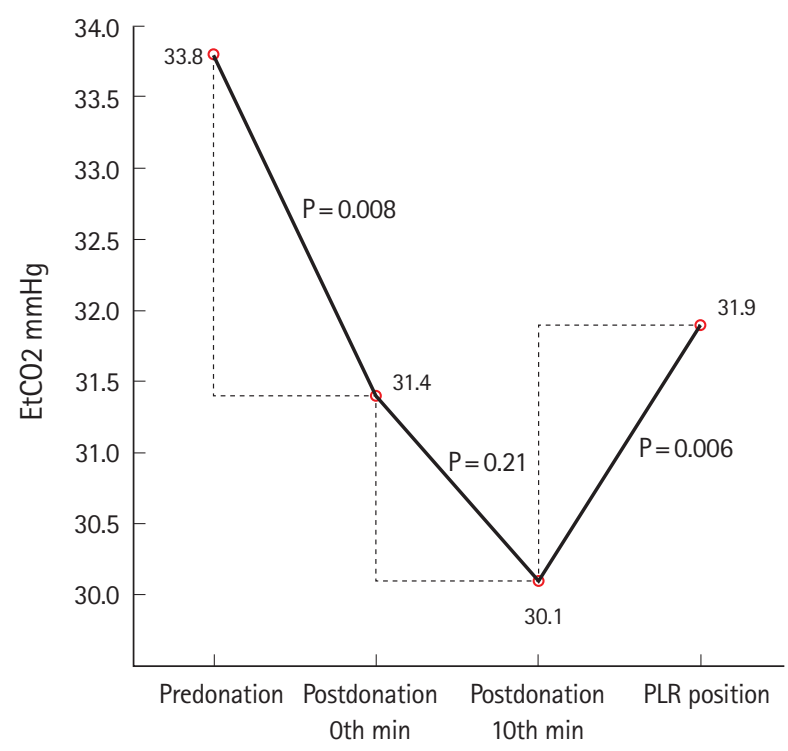

Fig. 3. Variations in end-tidal carbon dioxide (EtCO2). PLR, passive leg raise.

crease in the EtCO2 obtained in the PLR position compared with that obtained at the 10th min postdonation (MD, 1.8 $\pm 3.2,95 \%$ $\mathrm{Cl}, 0.074$ to $4.44, \mathrm{P}=0.006$ ) (Fig. 3). Compared with the predonation value, the MAP significantly decreased at the 10th minute postdonation (MD, 6.4 $\pm 6.4 ; 95 \% \mathrm{Cl}, 3$ to $9.7 ; \mathrm{P}<0.001$ ) (Fig. 4). There were no significant changes in the $\mathrm{VCCl}$ and $\mathrm{PP}$ values ( $P=1.0$ for both values).

The change directions of the EtCO2 and MAP were consistent for all measurements (Figs. 2, 3). Further, the change direction of the PVI was consistently opposed to that of the EtCO2 and MAP (Fig. 1). However, there was no significant correlation between

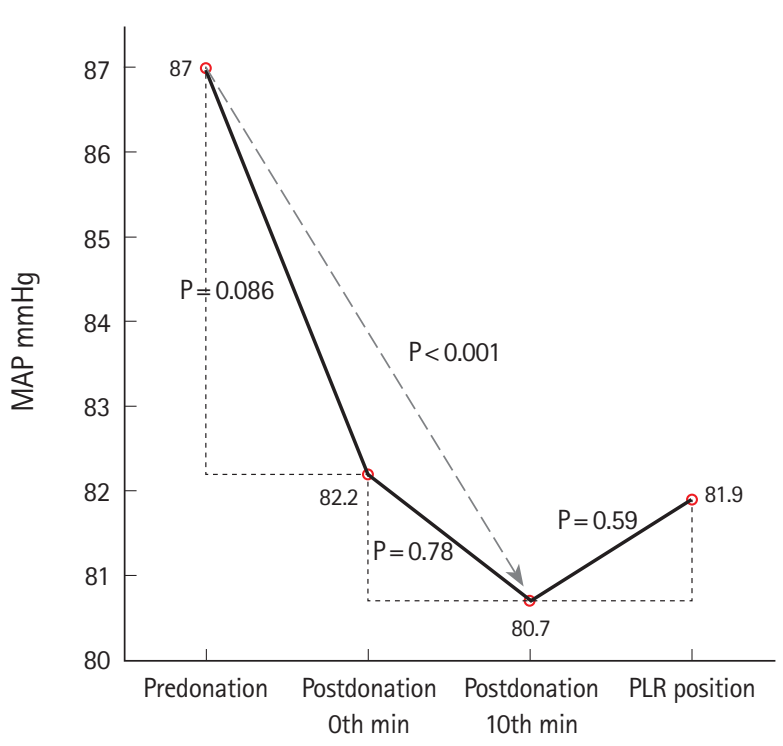

Fig. 4. Variations in mean arterial pressure (MAP). $P L R$, passive leg raise.

Table 2. Number of the patients showing a consistent direction of change between the measurements

\begin{tabular}{lll}
\hline & \multicolumn{1}{c}{ Blood donation } & \multicolumn{1}{c}{ PLR } \\
\hline PVI (direction of change) & 24 (80) (increase) & $16(53)$ (decrease) \\
EtCO2 (direction of change) & 20 (66) (decrease) & $19(63)$ (increase) \\
MAP (direction of change) & $16(53)$ (decrease) & 17 (56) (increase) \\
\hline
\end{tabular}

Values are presented as number (\%).

$\mathrm{PLR}$, passive leg raise; PVI, pleth variability index; EtCO2, end-tidal carbon dioxide; MAP, mean arterial pressure.

a) The measurement intervals in which statistically significant changes were detected in the post hoc analysis.

the $\mathrm{PVI}$ and $\mathrm{EtCO} 2$ changes between predonation and the 0th minute postdonation $(r=-0.168 ; P=0.375 ; 95 \% \mathrm{Cl},-0.447$ to $0.157)$ and between the 10th minute postdonation and PLR $(r=$ $-0.218 ; \mathrm{P}=0.246 ; 95 \% \mathrm{Cl},-0.563$ to 0.246 ). Table 2 presents the number of patients showing a consistent change direction of the $\mathrm{PVI}, \mathrm{EtCO}$, and MAP.

\section{DISCUSSION}

We observed that a 450-mL acute blood depletion led to a significant change in the PVI, EtCO2, and MAP. Moreover, there was a significant increase in EtCO2 in the PLR position. To our knowledge, this is the first study to report PVI changes with acute intravascular volume depletion in spontaneously breathing volunteers.

Previous studies have assessed the volume status and responsiveness in mechanically ventilated patients and have reported that dynamic indicators, including the PVI based on cardiopulmonary interactions, are the best guides. ${ }^{19,20}$ Although the utility of 
the PVI has been demonstrated in mechanically ventilated patients, its reliability in spontaneously breathing patients remains unclear. ${ }^{8,21}$ A recent study by Demirci et al. ${ }^{8}$ assessed PVI variations in the supine, Trendelenburg, and PLR positions in healthy spontaneously breathing volunteers. Although there was a significant change in the PVI, inconsistent change directions of the PVI indicated that it was not a reliable predictor of volume status change in those individuals. Schoonjans et al. ${ }^{10}$ assessed changes in the PVI and PP in hypovolemia related to hemodialysis and blood donation, as well as after PLR. There was a significant posthypovolemia change in the PVI (22\% vs. $18 \%, P=0.03)$; however, there was no significant post-PLR PP variation. Similarly, we observed a significant increase in the PVI after $450 \mathrm{~mL}$ blood loss; however, there was no significant change in consecutive PVI measurements (postdonation 10th minute and after PLR). There was a postdonation increase in the PVI of 24 (80\%) volunteers. This was consistent with the change direction of PVI in the entire study cohort. However, there were insignificant post-PLR changes in the PVI. Similar to the findings by Demirci et al., ${ }^{8}$ we observed an inconsistent post-PLR change in direction. This could be attributed to the fact that the volume expansion maneuvers, including PLR or Trendelenburg, could provide a fast transient change in the intravascular compartment without a significant change at the extravascular tissue level. Therefore, the PVI can better detect microcirculation changes at the tissue level after actual intravascular volume depletion.

Young et al. ${ }^{22}$ assessed the predictive ability of $\mathrm{EtCO} 2$ variation for volume responsiveness after PLR or $500 \mathrm{~mL}$ intravenous fluid bolus in mechanically ventilated patients in the intensive care unit. There was a significant increase in the delta EtCO2 in the volume responded group ( $5.9 \pm 7.6 \%$ vs. $1.4 \pm 4.4 \%, P=0.02)$. A similar study by Arango-Granados et al. ${ }^{12}$ evaluated the predictive ability of delta EtCO2 for PLR-induced volume responsiveness in spontaneously breathing volunteers and reported a weak correlation of PLR-induced changes in cardiac output (CO) with delta EtCO2. In our study, we observed a significant postdonation and post-PLR decrease and increase, respectively, in the mean EtCO2. This finding could be explained by the Frank-Starling Law, which implies a close correlation of $\mathrm{CO}$ and cardiac preload. ${ }^{23}$ Therefore, a decrease in the cardiac preload due to intravascular volume loss could result in a decreased $\mathrm{CO}$, which negatively affects adequate blood flow in tissue. Consequently, impaired microcirculation at the tissue level decreases metabolism and, in turn, EtCO2. On the other hand, a post-PLR increase in the CO improves microcirculation, which improves tissue metabolism and increases EtCO2.

Previous studies have assessed the utility of ultrasonographic- determined diameters of the inferior vena cava is assessing both volume status and responsiveness. ${ }^{16}$ However, inconsistent findings have rendered the reliability of $\mathrm{VCCl}$, especially in spontaneously breathing population, unclear. ${ }^{24}$ This inconsistency could be attributed to differences in the respiratory patterns in spontaneously breathing patients. Akilli et al. ${ }^{17}$ studied the inferior vena cava diameter as a hemorrhagic shock marker in spontaneously breathing patients and found that it was more valuable than traditional shock parameters, including the shock index, base excess, or lactic acid. In our study, there was no significant postdonation and post-PLR change in the $\mathrm{VCCl}$. Although the previous study reported that sonographic evaluation of the inferior vena cava could detect hemodynamic changes in patients with hemorrhagic shock, it might not be reliable in early stage shock.

Based on blood loss severity, hemorrhagic shock is graded as class 1 to $4 .{ }^{25}$ Class 1 shock is defined as minor blood loss $(<750$ $\mathrm{mL})$, which is not associated with significant vital sign changes. ${ }^{26}$ MAP and PP are derived from the arterial BP and are frequent targets for ensuring tissue perfusion. ${ }^{23}$ However, they rarely are affected in early phase hypovolemia. ${ }^{25}$ In our study, the volunteers can be considered as having postdonation class 1 shock. Although we observed a significant change in the systolic BP, class 1 shock does not usually involve a change in the systolic BP, MAP, heart rate, $\mathrm{PP}$, and respiratory rate. ${ }^{25,26}$ Our findings indicate that the PVI and EtCO2 could promptly detect acute $450 \mathrm{~mL}$ blood loss before the other vital parameters. Moreover, a majority of the population showed a consistent direction in the significant change. Therefore, PVI and EtCO2 could be paramount to monitoring patients with acute blood loss who have normal vital parameters.

This study has several limitations. First, our participants were all male since the majority of the blood donors in our center were male (96.5\%). Regarding critically ill patients in the ED, the heterogeneity of the study population limits the generalizability of the findings. For example, the pulse oximeter PPG waveform could be affected by systemic vascular resistance. Therefore, clinical conditions that affect systemic vascular resistance, including distributive shock or vasoactive drug use, might affect the reliability of PVI. Second, all our study participants had a hemoglobin level of $>13 \mathrm{~g} / \mathrm{dL}$. Therefore, our findings might be inapplicable to patients with anemia. Third, the utility of PVI in spontaneously breathing patients has not been validated by studies with larger study cohorts. Most previous studies on PVI have assessed mechanically ventilated patients with constant minute ventilation. Therefore, the reliability of PVI in spontaneously ventilating patients with varying respiratory patterns remains unclear. Nevertheless, since there is a relatively regular respiratory pattern in healthy volunteers, our findings increase the credibility of PVI utility in healthy 
spontaneously breathing individuals.

In conclusion, our findings indicate that the PVI and EtCO2 can detect hemodynamic changes after acute $450 \mathrm{~mL}$ blood loss in healthy volunteers. The change in direction was inversely consistent between PVI and EtCO2. However, their ability to guide volume status in spontaneously breathing patients remains unclear.

\section{CONFLICT OF INTEREST}

No potential conflict of interest relevant to this article was reported.

\section{REFERENCES}

1. Cecconi $M$, De Backer D, Antonelli $M$, et al. Consensus on circulatory shock and hemodynamic monitoring. Task force of the European Society of Intensive Care Medicine. Intensive Care Med 2014;40:1795-815.

2. Henning DJ, Kearney KE, Hall MK, Mahr C, Shapiro NI, Nichol G. Identification of hypotensive emergency department patients with cardiogenic etiologies. Shock 2018;49:131-6.

3. Boulain T, Cecconi M. Can one size fit all? The fine line between fluid overload and hypovolemia. Intensive Care Med 2015;41:544-6.

4. Alyesil C, Dogan NO, Ozturan IU, Guney S. Distributive Shock in the emergency department: sepsis, anaphylaxis, or capillary leak syndrome? J Emerg Med 2017;52:e229-31.

5. Marik PE. Techniques for assessment of intravascular volume in critically ill patients. J Intensive Care Med 2009;24:329-37.

6. Middleton PM, Davies SR. Noninvasive hemodynamic monitoring in the emergency department. Curr Opin Crit Care 2011; 17:342-50.

7. Takahashi S, Kakiuchi S, Nanba Y, Tsukamoto K, Nakamura T, Ito $Y$. The perfusion index derived from a pulse oximeter for predicting low superior vena cava flow in very low birth weight infants. J Perinatol 2010;30:265-9.

8. Demirci OL, Cikrikci Isik G, Corbacioglu SK, Cevik Y. Comparing Pleth variability index (PVI) variation induced by passive leg raising and Trendelenburg position in healthy volunteers. Am J Emerg Med 2020;38:278-81.

9. Liu T, Xu C, Wang M, Niu Z, Qi D. Reliability of pleth variability index in predicting preload responsiveness of mechanically ventilated patients under various conditions: a systematic review and meta-analysis. BMC Anesthesiol 2019;19:67.

10. Schoonjans $A$, Forget $P$, Labriola $L$, et al. Pleth variability index combined with passive leg raising-induced pulse pressure variation to detect hypovolemia in spontaneously breathing pa- tients. Acta Anaesthesiol Belg 2010;61:147-50.

11. Pekdemir M, Cinar O, Yilmaz S, Yaka E, Yuksel M. Disparity between mainstream and sidestream end-tidal carbon dioxide values and arterial carbon dioxide levels. Respir Care 2013;58: 1152-6.

12. Arango-Granados MC, Zarama Cordoba V, Castro Llanos AM, Bustamante Cristancho LA. Evaluation of end-tidal carbon dioxide gradient as a predictor of volume responsiveness in spontaneously breathing healthy adults. Intensive Care Med Exp 2018;6:21.

13. Kheng $\mathrm{CP}$, Rahman $\mathrm{NH}$. The use of end-tidal carbon dioxide monitoring in patients with hypotension in the emergency department. Int J Emerg Med 2012;5:31.

14. Freedman SB, Johnson DW, Nettel-Aguirre $A$, et al. Assessing dehydration employing end-tidal carbon dioxide in children with vomiting and diarrhea. Pediatr Emerg Care 2018;34:564-9.

15. Yang HW, Jeon W, Min YG, Lee JS. Usefulness of end-tidal carbon dioxide as an indicator of dehydration in pediatric emergency departments: a retrospective observational study. Medicine (Baltimore) 2017;96:e7881.

16. Dipti A, Soucy Z, Surana A, Chandra S. Role of inferior vena cava diameter in assessment of volume status: a meta-analysis. Am J Emerg Med 2012;30:1414-9.

17. Akilli $B$, Bayir $A$, Kara $F, A k A$, Cander B. Inferior vena cava diameter as a marker of early hemorrhagic shock: a comparative study. Ulus Travma Acil Cerrahi Derg 2010;16:113-8.

18. El Ayadi AM, Nathan HL, Seed PT, et al. Vital sign prediction of adverse maternal outcomes in women with hypovolemic shock: the role of shock index. PLoS One 2016;11:e0148729.

19. Cannesson M, Delannoy B, Morand A, et al. Does the Pleth variability index indicate the respiratory-induced variation in the plethysmogram and arterial pressure waveforms? Anesth Analg 2008;106:1189-94.

20. Michard F, Boussat $S$, Chemla D, et al. Relation between respiratory changes in arterial pulse pressure and fluid responsiveness in septic patients with acute circulatory failure. Am J Respir Crit Care Med 2000;162:134-8.

21. Cannesson $M$, Attof $Y$, Rosamel $P$, et al. Respiratory variations in pulse oximetry plethysmographic waveform amplitude to predict fluid responsiveness in the operating room. Anesthesiology 2007;106:1105-11.

22. Young A, Marik PE, Sibole $S$, Grooms D, Levitov A. Changes in end-tidal carbon dioxide and volumetric carbon dioxide as predictors of volume responsiveness in hemodynamically unstable patients. J Cardiothorac Vasc Anesth 2013;27:681-4.

23. Hallisey SD, Greenwood JC. Beyond mean arterial pressure and lactate: perfusion end points for managing the shocked 
patient. Emerg Med Clin North Am 2019;37:395-408.

24. Orso D, Paoli I, Piani T, Cilenti FL, Cristiani L, Guglielmo N. Accuracy of ultrasonographic measurements of inferior vena cava to determine fluid responsiveness: a systematic review and meta-analysis. J Intensive Care Med 2020;35:354-63.

25. Kobayashi L, Costantini TW, Coimbra R. Hypovolemic shock resuscitation. Surg Clin North Am 2012;92:1403-23.

26. Cannon JW. Hemorrhagic shock. N Engl J Med 2018;378:370-9. 